\title{
...hablando del derecho a la Educación Sexual Integral: la voz de los adolescentes en Ecuador
}

\section{... speaking about the right to Integral Sexuality} Education : the voice of adolescents in Ecuador

... falando sobre o direito à educação sexual integral: a voz do adolescentes no Equador

\section{Castillo Jéssica}

Universidad de Cuenca, Ecuador

E-mail: Jessica.castillo@ucuenca.edu.ec

\section{Derluyn Ilse}

Department of Social Work and Social Pedagogy, Ghent University, Gent,

$$
\text { Belgium }
$$

E-mail:

\section{Valcke Martin}

Department of Educational Studies, Ghent University, Gent, Belgium E-mail:

\section{Resumen}

En base a la premisa de la importancia de la voz de los adolescentes en el diseño de propuestas de educación sexual, el presente estudio explora las necesidades de los adolescentes de educación sexual con enfoque integral en el sistema educativo y sus percepciones respecto al desempeño de sus docentes en el tema, considerando diferencias de género. El estudio de corte cuantitativo involucró a 780 estudiantes entre 11 y 19 años 
de edad. El análisis descriptivo indicó que las necesidades de educación sexual son altas y la satisfacción con el desempeño de sus docentes es media. La regresión logística multinomial reveló que no existen diferencias significativas entre adolescentes varones y mujeres en cuanto a las necesidades de educación sexual pero sí en cuanto a la percepción del desempeño de sus docentes. Los resultados tienen claras implicaciones para el diseño e implementación de programas de educación sexual, y para fortalecer propuestas de formación docente aportando así, al empoderamiento del derecho a la educación sexual.

Palabras Clave: educación sexual integral, adolescentes, docentes, Ecuador

\section{Abstract}

Grounded on the premise of the importance of adolescents' voice in sexuality education proposals design, this study explores adolescents' needs of sexuality education from a comprehensive approach in the education system, and their perceptions regarding their teachers' performance in the topic, considering gender differences. The quantitative study involved 780 students between 11 and 19 years old. The descriptive analysis indicated that the needs of sexual education are high and the satisfaction with the performance of their teachers is middle. The multinomial logistic regression revealed that there are no significant differences between male and female adolescents in terms of sexuality education needs, but there are in terms of the perception of their teachers' performance. The results have clear implications for the design and implementation of sexuality education programs, and to strengthen proposals for teacher training thus contributing to the empowerment of sexuality education right.
Key words: comprehensive sexual education, adolescents, teachers, Ecuador

\section{Resumo}

Com base na premissa da importância da voz dos adolescentes no projeto de propostas de educação sexual, este estudo explora as necessidades dos adolescentes na educação sexual com uma abordagem abrangente do sistema educacional e suas percepções sobre o desempenho de seus professores no assunto, considerando as diferenças de gênero. $\mathrm{O}$ estudo de corte quantitativo envolveu 780 estudantes entre 11 e 19 anos de idade. A análise descritiva indicou que as necessidades da educação sexual são altas e a satisfação com o desempenho de seus professores é média. A regressão logística multinomial revelou que não há diferenças significativas entre adolescentes do sexo masculino e feminino em termos de necessidades de educação sexual, mas em termos de percepção do desempenho de seus professores. Os resultados têm implicações claras para a concepção e implementação de programas de educação sexual e para fortalecer as propostas de formação de professores, contribuindo assim para o empoderamento do direito à educação sexual.

Palavras-chave: educação sexual abrangente, adolescentes, professores, Equador 


\section{Introducción*18}

Actualmente y a nivel mundial, cada vez más los adolescentes son reconocidos como sujetos sexuados y, por lo tanto, con el derecho primordial de acceder a una educación de la sexualidad y salud sexual y reproductiva basada en fundamentos científicos y sensible a realidades socio-culturales (Allen, 2005; Santelli et al., 2006; Haberland \& Rogow, 2015; UNESCO, 2015). Así, la educación sexual para la juventud es crucial para su bienestar y desarrollo.

A pesar de no existir un consenso generalizado respecto a los mecanismos mas efectivos para lograr la promociòn y empoderamiento de los derechos sexuales y reproductivos en los adolescentes (Hirst, 2008; Denno, Hoopes, \& Chandra-Mouli, 2015), existe evidencia científica que apoya el hecho de que el desarrollo o mejora de propuestas de educación sexual, deben partir de los intereses y necesidades de los distintos grupos de adolescentes y sus particularidades (Allen, 2005; Helmer, Senior, Davison, \& Vodic, 2015)

En este sentido, la relevancia de la participación de los adolescentes en el desarrollo de propuestas educativas relacionadas a educación sexual es reconocida (Kirby, 2002; Forrest, Strange, Oakley, \& Ripple Study Team, 2004), pero su voz es casi ausente, sino nula en este escenario. En la gran mayoría de los casos, son los adultos quienes deciden las necesidades e intereses de educación sexual de los adolescentes y quienes, por lo tanto, diseñan propuestas educativas en la temática frecuentemente en base a estándares predefinidos en otros contextos (Santelli et al., 2006). Sin embargo, no queda claro si propuestas desarrolladas de esta manera, responden a las necesidades e intereses de los mismos adolescentes (Forrest et al., 2004).

$18{ }^{\star}$ Agradecemos al Programa de Cooperación entre la Universidad de Cuenca y las Universidades Flamencas (VLIR-UOS) que auspició el presente estudio.
Un espacio estratégico, que se ha visto influenciado por esta mirada adulto-céntrica de las necesidades de educación sexual de los adolescentes, es el sistema educativo formal y sus propuestas en la temática. Si bien, el sistema educativo formal es reconocido como clave para promover el derecho al acceso a una educación sexual integral (UNESCO, 2015), también la escuela y/o colegio se han visto en la disyuntiva de buscar formas de implementar un tema con una larga trayectoria de controversias (Bay-Cheng, 2003), especialmente en contextos como el ecuatoriano.

A pesar de las inminentes dificultades de implementar la educación sexual en el sistema educativo, el potencial de la institución educativa formal es claro pues, constituye un ambiente relativamente seguro y confiable para abordar el tópico y, permite llegar a un grupo importante adolescentes de diferentes contextos (Thomas \& Aggleton, 2016). Además, existe evidencia de que los mismos adolescentes reconocen la importancia del rol del colegio en la provisión de educación sexual (Walters \& Hayes, 2007).

Es reconocido, tanto a nivel mundial como latinoamericano, que muchos sistemas educativos formales, abordan la educación sexual; sin embargo, la participación de los niños, niñas y adolescentes escolarizados en su diseño es exigua (Bay-Cheng, 2003; MacDonald et al., 2011). Esta situación es preocupante si se considera, como se mencionó anteriormente, que abarcar las perspectivas del grupo cuyas necesidades e intereses aspiran a ser atendidas en las escuelas y colegios, es crucial para el involucramiento de los estudiantes en el currículo de educación sexual (Allen, 2008); además que se les visibiliza como sujetos sexuados y con derechos (Santelli et al., 2006; Haberland \& Rogow, 2015).

La Literatura científica en torno a programas de educación sexual en escuelas o colegios, se ha centrado más en los resultados luego de implementar un programa respectivo (Suellentrop, 2011); o en obstáculos en la implementación (Simovska \& Kane, 2015). Además, varios estudios 
tienen más un acercamiento a un enfoque preventivo de riesgos más que a otros enfoques que vayan más allá de esta visión negativa de la sexualidad, como lo es el enfoque integral por ejemplo (Kirby, 2002; Rijsdijk, Lie, Bos, Leerlooijer, \& Kok, 2013).

La falta de estudios investigando las necesidades e intereses de los niños, niñas y adolescentes respecto a un enfoque integral de sexualidad en los colegios, es más evidente en países de bajos y medianos ingresos. Es en estos países, donde la salud sexual y reproductiva de los adolescentes es pobre (Patton et al., 2012); se presentan altas tasas de embarazo adolescente, de violencia de género, y contagios de SIDA, junto a una debilidad general de programas educativos centrados en adolescentes (Chandra-Mouli et al., 2015).

Esta situación es también evidente en el contexto ecuatoriano. Ecuador reflejó una de las tasas más altas de embarazo adolescentes en Latino América en 2010 (MSE, 2012) con un alto crecimiento de embarazo en adolescentes a cada vez, más temprana edad; además, una alta prevalencia de violencia de género (INEC, 2014). De hecho, en el contexto ecuatoriano, las diferencias de género se encuentran frecuentemente asociadas a inequidad, subordinación, discriminación y heteronormatividad (Viveros, 2010). Estos hechos confirmar, entre otros aspectos, las limitaciones en los programas de educación sexual en el Ecuador.

En el presente estudio, en base a la premisa de escuchar la voz a los adolescentes respecto a sus necesidades e intereses en cuanto a la educación sexual, se busca explorar las percepciones de los adolescentes respecto a sus necesidades de educación sexual en el colegio desde un enfoque integral y su posible asociación con el género de los adolescentes. Así mismo, se pretende examinar las percepciones de los adolescentes respecto al desempeño de sus docentes en la temática de educación sexual y también, su posible asociación con el género de los adolescentes.

\subsection{Educación Sexual Integral}

La educación sexual integral u holística, refleja una visión integral de la sexualidad humana. Esta se fundamenta en un enfoque de educación sexual basado en derechos y centrado en el género como lo promueve la UNESCO (2009) y el UNFPA (2014). Así, la educación sexual integral sostiene que se deben abordar diferentes dimensiones, incluida la vida familiar, las relaciones, la cultura, los roles de género y la violencia sexual:

"La educación integral en sexualidad busca equipar a los jóvenes con el conocimiento, las habilidades, las actitudes y los valores que necesitan para determinar y disfrutar su sexualidad, física y emocional, individualmente y en las relaciones. Considera la sexualidad de manera holística y en el contexto del desarrollo emocional y social. Reconoce que la información sola no es suficiente. Los jóvenes deben tener la oportunidad de adquirir habilidades esenciales para la vida y desarrollar actitudes y valores positivos. La educación sexual integral debe ayudar a los jóvenes a adquirir información precisa, desarrollar habilidades para la vida y alimentar actitudes y valores positivos " (IPPF, 2006 p. 6).

Así, la educación sexual integral debe ser apropiada para la edad y coherente con las capacidades en evolución de los jóvenes, debe ser culturalmente relevante y científicamente precisa, y debe proporcionar información realista y sin prejuicios. Además, la educación sexual integral no solo debe pasar información, sino que debe generar oportunidades para explorar sus propios valores y actitudes y, para construir habilidades de toma de decisiones, comunicación y reducción de riesgos con respecto a la sexualidad.

En el presente estudio, se ha considerado el marco de la educación sexual integral propuesto por la IPPF (2006) que abarca siete dimensiones: roles de género, salud sexual, derechos sexuales, placer, violencia, diversidad y relaciones. 


\subsection{Educación sexual integral en el contexto ecuatoriano}

En Ecuador, ha habido continuas modificaciones en la visión educativa sobre la educación sexual (MEE, 2012). Hace unos 6 años, en Ecuador se adoptó un enfoque de Educación Integral de la Sexualidad como una parte obligatoria del currículo escolar general. Además, la educación sexual integral se estableció como un eje transversal dentro del currículo escolar. Esto implicaba que cada maestro, en cualquier nivel educativo y responsable de cualquier asignatura, debe ser capaz de abordar la educación sexual en su aula. A pesar de estas regulaciones de política, hubo una falta de implementación sistemática de este programa además de ningún seguimiento.

En el año 2016, la educación sexual en el Ecuador tuvo un retroceso con el establecimiento del "Plan Familia" que retomaba valores tradicionales de la familia como el marco de abordaje de la sexualidad de los adolescentes (CGPGEE, 2016). En mayo de 2017, el plan familia fue cancelado (Carvajal, 2017) y las políticas de educación sexual han dado un giro con la propuesta de 3 proyectos de ley que buscan fortalecer la educación sexual con un enfoque de género. Lamentablemente la información sobre esta propuesta ha sido tergiversada, recibiendo el rechazo de gran parte de la población.

Hasta el momento, hay muy poca evidencia empírica sobre la naturaleza y el impacto de las iniciativas sobre programas de educación sexual en las escuelas y colegios ecuatorianos (ver, por ejemplo, Pineda \& Jerves, 2015). Los datos disponibles reflejan un desajuste entre las regulaciones nacionales para la educación sexual en las instituciones educativas y las disposiciones reales en las escuelas (Ortiz \& Palacios, 2009). Esta situación es alarmante ya que Ecuador tiene una de las tasas más altas de embarazo temprano en América Latina (Lora Rocha, Castro Mantilla, \& Salinas Mulder, 2009; MSE, 2012)

\section{Métodos}

El estudio fue de corte cuantitativo transversal, nivel exploratorio.

\subsection{Participantes}

Los participantes fueron estudiantes adolescentes de Unidades Educativas (UE) y Colegios ${ }^{19}$ fiscales mixtos de áreas urbanas, semiurbanas y rurales de la provincia del Azuay. Los participantes fueron seleccionados mediante un proceso de muestreo estratificado en múltiples etapas:

En un primer momento, en base a la información demográfica de la provincia del Azuay (INEC, 2010), ocho cantones fueron seleccionados (50\% de todos los cantones). Seguidamente, revisando la base de datos de las escuelas públicas de Azuay, $18 \mathrm{UE/Colegios} \mathrm{fueron} \mathrm{seleccionados}$ al azar en estos ocho cantones: de cinco cantones: uno urbano y otro rural (diez en total); de dos cantones rurales: una UE/Colegio de cada cantón (dos en total); y del cantón Cuenca, dos UE/Colegios urbanos, dos rurales y dos urbano-marginales (seis en total).

En un segundo momento, se consideraron tres categorías de grupos de estudiantes en cada UE/Colegio seleccionado. Grupo 1: estudiantes de $8^{\text {vo }}$ a $9^{\text {no }}$ de básica (adolescencia temprana); grupo 2: estudiantes de $10^{\text {mo }}$ de básica y de $1^{\mathrm{er}}$ año bachillerato (adolescencia intermedia); y grupo 3 : estudiantes de $2^{\text {do }}$ y $3^{\text {er }}$ año de bachillerato (adolescencia tardía).

Finalmente, en un tercer momento, un grupo de al menos 15 (5\% error muestral) estudiantes fue seleccionado al azar de cada categoría explicada anteriormente, considerando un balance entre hombres y mujeres. La muestra total estuvo constituida por 780 adolescentes entre 10 y 19

19 Puesto que el levantamiento de datos para el presente estudio se realizó en un momento de transición de los Colegios Secundarios a Unidades Educativas, se consideraron Unidades Educativas o Colegios que tenían estudiantes de entre 11 y 19 años en la misma institución. 
años. De este grupo, $\mathrm{N}=398$ (51.1\%) adolescentes fueron varones y $\mathrm{N}=$ $382(48.9 \%)$ fueron mujeres. Para el caso de la segunda escala, se tomó en cuenta solo los adolescentes que reportaron haber recibido educación sexual. Así, 702 adolescentes, de los cuales 354 (50.4\%) varones y 348 $(49.6 \%)$ mujeres, reportaron haber tenido educación sexual en sus colegios. Esta muestra refleja las características demográficas de la población del Azuay en términos género de los adolescentes (INEC, 2010).

\subsection{Procedimiento}

Después de obtener la respectiva autorización de parte de las autoridades locales del Ministerio de Educación, las UC/Colegios seleccionados fueron visitadas. Los rectores de las UE/Colegios después de verificar la respectiva carta de autorización permitieron a los encuestadores capacitados para el efecto, aplicar el instrumento a los participantes. Los encuestadores, conjuntamente con los inspectores/profesores de los colegios, seleccionaron al azar los tres grupos de adolescentes en cada colegio en base a una revisión y selección de los respectivos listados de estudiantes. La aplicación de la encuesta empezó indicando a los adolescentes acerca de los objetivos del estudio, luego se revisó y firmó un consentimiento informado. Todos los adolescentes seleccionados, aceptaron participar en el estudio. Después de llenar la encuesta los adolescentes participantes recibieron un snack.

\subsection{Instrumento}

Para llevar a cabo el estudio se desarrolló un cuestionario. Aparte de una sección inicial para obtener información sociodemográfica. el cuestionario estuvo compuesto por dos escalas.

La primera escala evaluó las percepciones de las necesidades de educación sexual integral de los adolescentes. La escala estuvo constituida por 42 ítems: 6 ítems por cada una de las siete dimensiones de la educación sexual integral propuestas por la IPPF (IPPF, 2006; IPPF, 2012; WHO, 2010).

Las siete dimensiones evaluadas fueron: roles de género, salud sexual, derechos sexuales, placer, violencia, diversidades y relaciones. La escala fue de tipo Likert con valores del 1 al 6 para indicar en qué medida cada ítem era considerado como más o menos importante aprender a los adolescentes; donde 1 fue el valor menos importante y 6 el más importante. Ejemplo de item: dimensión salud sexual "evitar embarazos no deseados".

La segunda escala evaluó las percepciones de los adolescentes respecto al desempeño de sus profesores en el tema de educación sexual. La escala estuvo constituida por 6 ítems y fue desarrollada en base a Bandura (2006) and (WHO, 2010). La escala también fue de tipo Likert con valores del 1 al 6 para indicar en qué medida los adolescentes estaban de acuerdo con el desempeño de sus docentes; donde 1 fue el valor de menos acuerdo y 6 el de mayor acuerdo. Ejemplo de ítem: "mis profesores nos pueden motivar a sentirnos cómodos mientras abordamos temas relacionados con educación sexual".

El cuestionario fue sometido a un proceso previo de validación a través de la revisión de expertos y una prueba piloto. La prueba piloto fue con una muestra de estudiantes distinta a la del estudio definitivo, pero con similares características. Los índices de confiablidad de Cronbach del cuestionario final son aceptables al ser mayores a $\mathrm{a}=0.70$ en todas las escalas.

\subsection{Análisis de datos}

Siendo un estudio exploratorio, se inició con un análisis descriptivo. Puesto que los datos reflejaron en general resultados altos, la escala original fue colapsada y recodificada en tres nuevos valores: 1,2 y 3 . 
Siendo 1 el valor mínimo y 3 el valor máximo. Este tipo de procedimiento es usado con frecuencia en investigaciones que involucran temas sensibles, cuando los participantes seleccionan un rango pequeño de valores dentro de una escala más amplia (ver e.g., Matthias, Lubben, Atchison, \& Schweitzer, 1997). Se aplicó la guía de Linacre (2002) para colapsar los valores iniciales y obtener las nuevas categorías de respuestas.

Los porcentajes presentados fueron calculados en base a las escalas re categorizadas. Posteriormente, también con las escalas recategorizadas, se realizó un análisis inferencial. Se aplicó una regresión logística multinomial para establecer diferencias de las variables de estudio en relación al género de los participantes. En el modelo de regresión, el género de los adolescentes fue ingresado como predictor de la importancia dada a los puntajes de las siete dimensiones de la educación sexual en un primer momento. En un segundo momento, el género fue ingresado como predictor del acuerdo con el desempeño de los docentes en educación sexual.

Antes de ejecutar la regresión logística multinomial, fue examinada la homogeneidad de varianzas. El test de Levene reveló que el supuesto de la homogeneidad de varianzas fue consistentemente logrado. El nivel de significancia fue establecido a .05 y el análisis se desarrolló usando el SPSS 19.0.

\section{Resultados}

El objetivo del presente estudio fue explorar las percepciones de los adolescentes varones y mujeres respecto a a) sus necesidades de educación sexual con un enfoque integral en el colegio b) el desempeño de sus docentes en la temática de educación sexual

En cuanto a las necesidades de educación sexual, los resultados indican que los adolescentes expresan tener necesidades muy altas de educa- ción sexual en todas las dimensiones evaluadas (Tabla1) . Esto es especialmente notorio al considerar los valores 2 (importante) y 3 (muy importante) juntos los cuales son mucho más altos que el valor 1 (menos importante). La tendencia general indica necesidades más altas en las dimensiones de salud sexual y violencia; mientras que las necesidades más bajas son en la dimensión de placer.

Tabla 1. Necesidades de los adolescentes de educación sexual con enfoque integral según género $(\mathrm{N}=780)$

\begin{tabular}{l|ccc|ccc|ccc}
\hline & \multicolumn{3}{|c|}{$\begin{array}{c}\text { Roles de } \\
\text { Género }\end{array}$} & \multicolumn{2}{|c|}{ Salud Sexual } & \multicolumn{3}{|c}{$\begin{array}{c}\text { Derechos } \\
\text { Sexuales }\end{array}$} \\
\hline \multicolumn{1}{c|}{$\begin{array}{l}\text { Valores } \\
\text { Género }\end{array}$} & $\mathbf{1}$ & $\mathbf{2}$ & $\mathbf{3}$ & $\mathbf{1}$ & $\mathbf{2}$ & $\mathbf{3}$ & $\mathbf{1}$ & $\mathbf{2}$ & $\mathbf{3}$ \\
Hombres (\%) & 35.9 & 40.2 & 23.8 & 20.1 & 35.1 & 44.7 & 21.8 & 42.4 & 35.6 \\
Mujeres (\%) & 30.1 & 39.8 & 30.1 & 13.6 & 32.4 & 54.7 & 21.2 & 38.7 & 40.5 \\
\hline
\end{tabular}

\begin{tabular}{l|ccc|ccc|ccc|ccc}
\hline & \multicolumn{3}{|c|}{ Placer } & \multicolumn{3}{|c|}{ Violencia } & \multicolumn{2}{c|}{ Diversidad } & \multicolumn{3}{|c}{ Relaciones } \\
\hline \multicolumn{1}{c|}{$\begin{array}{l}\text { Valores } \\
\begin{array}{l}\text { Género } \\
\text { Hombres (\%) }\end{array}\end{array}$} & $\mathbf{1}$ & $\mathbf{2}$ & $\mathbf{3}$ & $\mathbf{1}$ & $\mathbf{2}$ & $\mathbf{3}$ & $\mathbf{1}$ & $\mathbf{2}$ & $\mathbf{3}$ & $\mathbf{1}$ & $\mathbf{2}$ & $\mathbf{3}$ \\
Mujeres (\%) & 36.1 & 42.7 & 21.1 & 23.1 & 28.6 & 48.2 & 25.4 & 41.2 & 33.3 & 30.9 & 41.2 & 27.8 \\
& 35.6 & 41.0 & 23.2 & 18.8 & 21.7 & 59.4 & 23.0 & 33.2 & 43.7 & 28.7 & 40.1 & 31.1 \\
\hline
\end{tabular}

Nota: $1=$ Menos importante; 2 = Importante; 3 = Muy importante.

Los resultados de la regresión logística multinomial para las necesidades de educación sexual se presentan en la tabla 2. Según indican los resultados, la variable género no indica asociación significativa con ninguna de las siete dimensiones de la educación sexual integral. 
Tabla 2. Regresión logística multinomial para las dimensiones de educación sexual integral asociadas a las necesidades de educación sexual por género

\begin{tabular}{|c|c|c|c|c|c|c|c|c|c|}
\hline \multirow[b]{2}{*}{$\mathbf{b}(\mathbf{S E})^{\phi}$} & \multicolumn{2}{|c|}{ Roles de Género $^{\Pi}$} & \multicolumn{3}{|c|}{ Salud Sexual } & \multicolumn{3}{|c|}{$\begin{array}{l}\text { Derechos } \\
\text { Sexuales }\end{array}$} & \\
\hline & $\begin{array}{l}\text { Odds } \\
\text { Ratio }\end{array}$ & b(SE) & Odds Ra & atio & $\mathbf{b}(\mathbf{S E})$ & & $\begin{array}{l}\text { Odds } \\
\text { Ratio }\end{array}$ & b(SE) & \\
\hline \multicolumn{10}{|c|}{$\begin{array}{l}\text { Menos importante (1) vs. muy } \\
\text { importante (3) }\end{array}$} \\
\hline \multicolumn{10}{|l|}{ Gender $^{\theta}$} \\
\hline Female & $-0.39(0.19)$ & 0.68 & $-0.58(0.2$ & & 0.55 & -0.0 & $9(0.20)$ & 0.91 & \\
\hline \multicolumn{10}{|c|}{ Importante (2) vs. muy important (3) } \\
\hline \multicolumn{10}{|l|}{$\begin{array}{l}\text { Gender } \\
\text { Female }\end{array}$} \\
\hline \multirow[b]{2}{*}{$\mathbf{b}(\mathbf{S E})^{\phi}$} & \multicolumn{2}{|c|}{ Placer } & \multicolumn{2}{|c|}{ Violencia } & \multicolumn{3}{|c|}{ Diversidad } & \multicolumn{2}{|c|}{ Relaciones } \\
\hline & $\begin{array}{l}\text { Odds } \\
\text { Ratio }\end{array}$ & b(SE) & $\begin{array}{l}\text { Odds } \\
\text { Ratio }\end{array}$ & $\mathbf{b}(\mathrm{SE})$ & $\begin{array}{l}\text { Od } \\
\text { Rat }\end{array}$ & & $\mathbf{b}(\mathrm{SE})$ & $\begin{array}{l}\text { Odds } \\
\text { Ratio }\end{array}$ & \\
\hline \multicolumn{10}{|c|}{$\begin{array}{l}\text { Menos importante (1) vs. } \\
\text { muy importante (3) }\end{array}$} \\
\hline \multicolumn{10}{|l|}{ Gender $^{\theta}$} \\
\hline \multicolumn{10}{|c|}{ Importante (2) vs. muy important (3) } \\
\hline $\begin{array}{l}\text { Gender } \\
\text { Female }\end{array}$ & $-0.06(0.19)$ & 0.93 & $-0.45(0.17)$ & 0.63 & $-0.51(($ & .17) & 0.59 & $-0.11(0.17)$ & 0.89 \\
\hline
\end{tabular}

$* * * \mathrm{p}<.001$

${ }^{11}$ Categorìa de referencia $=$ último valor

$\phi$ Valor de beta y error standard

${ }^{\oplus}$ Referencia: Hombre

Respecto a las percepciones de los adolescentes en cuanto al desempeño de sus docentes en la temática de educación sexual, el porcentaje más alto de adolescentes expresó estar medianamente de acuerdo con el desempeño de sus docentes en educación sexual (tabla 3 ), pero con pequeñas diferencias en porcentajes respecto a menos acuerdo y acuerdo muy alto.
Tabla 3. Satisfacción de los adolescentes con el desempeño de sus docentes en el tema de educación sexual $(\mathrm{N}=702)$

\begin{tabular}{llccc}
\hline & & \multicolumn{3}{c}{ Acuerdo con el desempeño de docentes en ES } \\
\hline \multirow{2}{*}{ Género } & Valores & $\mathbf{1}$ & $\mathbf{2}$ & $\mathbf{3}$ \\
& & & & \\
& Hombres (\%) & 34.4 & 35.3 & 30.2 \\
& Mujeres (\%) & 26.1 & 37.3 & 35.9 \\
\hline
\end{tabular}

Nota: $1=$ Menos acuerdo; 2 = acuerdo mediano; 3 = acuerdo muy alto.

La regresión logística multinomial indicó una asociación significativa entre las percepciones de los adolescentes respecto al desempeño docente en educación sexual y el género de los adolescentes. Así, las adolescentes mujeres estuvieron 1.50 veces más medianamente de acuerdo con el desempeño de sus docentes en educación sexual en comparación con los hombres. Estos resultados se pueden ver en la tabla 4.

Tabla 4. Regresión logística multinomial para el acuerdo con el desempeño docente en educación sexual por género $(\mathrm{N}=702)$

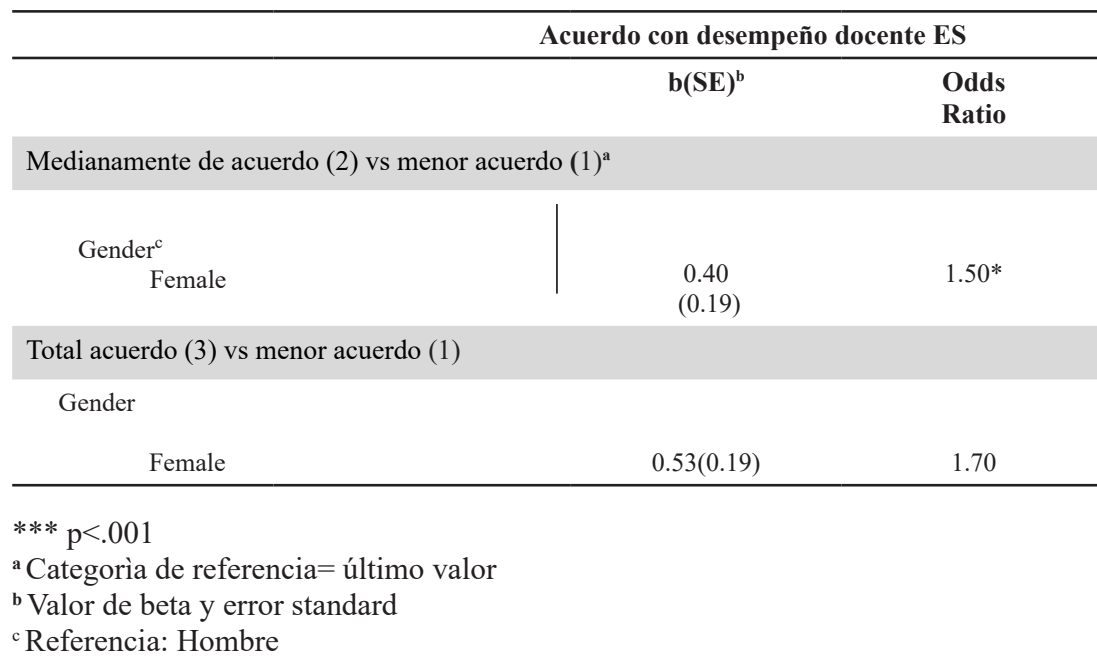




\section{Discusión}

Los resultados del presente estudio revelan que, en general, los adolescentes ecuatorianos de la provincia del Azuay expresan a) necesidades altas de educación sexual desde un enfoque integral y b) satisfacción media con el desempeño de sus docentes en la temática. Estos resultados indican que el sistema educativo formal no constituye un espacio que provea de la educación sexual que responda a los intereses y necesidades de los adolescentes. Así mismo, los resultados sugieren la necesidad de revisar latentes encuentros y desencuentros entre la educación sexual que actualmente reciben los adolescentes y sus necesidades expresadas.

Los hallazgos de este estudio además insinúan que los adolescentes tienen necesidades más maduras, complejas, amplias y diversas como ya lo ha indicado Allen (2008). Esta situación es particularmente interesante en contextos latinoamericanos donde suele prevalecer un enfoque negativo de educación sexual, basado en la salud y el riesgo (Darré, Jerves, Castillo, \& Enzlin, 2015). La visión medicalizada y de riesgos limita a los adolescentes en su posibilidad de abordar la sexualidad en todas sus dimensiones. Afecta también la toma de decisiones conscientes y responsables en su vida sexual, aspectos que constituyen un derecho fundamental de esa etapa de sus vidas.

En términos de las necesidades de educación sexual de los adolescentes, los hallazgos del presente estudio, concuerdan con reportes de trabajos previos que indican que, desde el punto de vista de los adolescentes, la educación sexual debería enfatizar un enfoque integral y, más específicamente, debería usar un marco más amplio que únicamente un enfoque sobre las consecuencias negativas de la actividad sexual (Allen, 2005; O’Higgins \& Gabhainn, 2010) o en una dimensión particular de la sexualidad, como su base biológica (Rijsdijk et al., 2013).

Al mirar los resultados porcentuales, las adolescentes mujeres expresaron mayores necesidades de educación sexual en comparación con los adolescentes varones. Esto coincide con estudios previos que sostienen mayores preocupaciones de las adolescentes mujeres en torno a su sexualidad y salud sexual (Forrest et al., 2004; O’Higgins \& Gabhainn, 2010). Sin embargo, el análisis inferencial no evidenció diferencias estadísticamente significativas entre las necesidades de adolescentes hombre y mujeres. En general, interpretando estos resultados dentro de la realidad de contexto ecuatoriano, se podría sugerir un par de hipótesis para explicar los resultados. Primeramente, al parecer hay una particular falta de empoderamiento de los derechos sexuales de las adolescentes mujeres. En segundo lugar, se podría sugerir una tendencia a "normalizar" las actuales normas de género presentes en la sociedad ecuatoriana. Ambos aspectos definitivamente requieren mayor investigación.

Los resultados de las percepciones de los adolescentes respecto al desempeño de sus docentes difieren en relación a estudios previos. Investigaciones en el área han documentado que los estudiantes no están satisfechos con la manera como sus maestros manejan la educación sexual por un lado y, consideran que sus profesores necesitan mejorar sus conocimientos, habilidades, actitudes y métodos de enseñanza, por otro lado (Hilton, 2003; Allen, 2005; Muhanguzi \& Ninsiima, 2011).

Estos resultados se podrían explicar considerando que el involucramiento de los estudiantes con el aprendizaje está influenciado por dinámicas culturales más amplias (Adams Tucker, George, Reardon, \& Panday, 2016). Puesto que los valores culturales a menudo se legitiman a través de mecanismos institucionales que resultan en códigos de práctica para docentes y profesionales de la salud (Shoveller, Johnson, Langille, \& Mitchell, 2004), es importante considerar las dinámicas sociales específicas del contexto de estudio. Hasta la fecha, los valores religiosos tradicionales son todavía muy importantes en Ecuador. Esto significa que es aceptable abordar temas relacionados a la anatomía, biología, reproducción y enfermedades de transmisión sexual. Pero, temas que vayan más allá de esta perspectiva biológica son difíciles de abordar. Entonces, estos resultados podrían sugerir que los adolescentes ecuatorianos podrían 
considerar que requieren menos en términos de educación sexual y sus requerimientos, y estos, al ser menores, serían satisfechos más pronto. Estas hipótesis requieren mayor investigación.

Complementariamente, los resultados acerca del menor nivel de satisfacción de los adolescentes varones con el desempeño de sus docentes en educación sexual son consistentes con estudios previos (Lupton \& Tulloch, 1996; Hilton, 2003; Muhanguzi \& Ninsiima, 2011). Estos hallazgos pueden también ser explicados por patrones culturales influenciando el comportamiento sexual de adolescentes varones y mujeres. Esto es, los adolescentes están expuestos a cierta información y viven determinadas experiencias respecto a las distintas dimensiones de la sexualidad (Allen, 2005). En este sentido, (Measor, 2004) indica que los adolescentes varones aprenden más acerca de la sexualidad excluyendo a los adultos (es decir adquieren mayor educación de los medios de comunicación y pares). Esta situación puede crear tensiones entre el contenido de educación sexual que aprenden y la forma como sus profesores lo manejan en los colegios y UE.

Los hallazgos de este estudio tienen algunas implicaciones. En primer lugar, es necesario conocer e incluir las necesidades e intereses autopercibidos en programas de educación sexual. Estos pueden proporcionar una base sólida sobre cómo las culturas locales están influyendo en la sexualidad de los adolescentes. Los programas de educación sexual serán mucho más eficaces cuando se consideren estas necesidades "locales" autopercibidas en su diseño, en combinación con ciertos temas centrales que se consideran esenciales para mantener una cierta calidad en el programa y para cumplir con los estándares internacionales (UNESCO, 2015). Además, este estudio insta a escuchar las voces de los adolescentes, ya que esto los reconoce como sujetos sexuales y aumenta el ejercicio de sus derechos. Respetar los puntos de vista de los adolescentes sobre las necesidades de educación sexual también puede ayudar a mejorar su bienestar sexual (MacDonald et al., 2011). Finalmente, para poder considerar y respetar las perspectivas de los adolescentes en el plan de estudios, se requiere un mayor desarrollo profesional de los docentes.
En último lugar, algunas limitaciones del presente estudio se deben considerar. Primeramente, los resultados están relacionados con un contexto ecuatoriano en particular, los resultados pueden no ser aplicables a otras regiones del Ecuador. Se necesita más investigación sobre las necesidades de sexualidad de los adolescentes en otras regiones de Ecuador y otros países andinos.

En segundo lugar, el estudio solo involucró a estudiantes de UE/Colegios secundarios públicas, a los que asisten predominantemente adolescentes con un estatus socioeconómico bajo y medio. Los resultados podrían variar al considerar estudiantes de colegios privados, sobre todo aquellos que pertenecen a órdenes religiosas. Los colegios privados pueden tener otras iniciativas de educación sexual para sus estudiantes.

Finalmente, dentro de la complejidad de las investigaciones asociadas a la sexualidad y educación sexual, es importante considerar que podría ser difícil para los adolescentes expresar sus necesidades reales con respecto a la educación sexual. Para ello, se recomendaría complementar la investigación utilizando otros tipos de recopilación de datos para confirmar nuestros hallazgos. Un estudio cualitativo podría proporcionar información más profunda respecto a las necesidades de educación sexual de los adolescentes y de cómo estas podrían ser satisfechas. Así mismo podría conocerse más sobre las eventuales barreras que los adolescentes experimentan para el empoderamiento de sus derechos.

\section{Conclusión}

La real implementación de la educación sexual desde un enfoque integral debe considerarse si se busca atender a las necesidades e intereses de los adolescentes de la región de estudio y aportar a mejorar su educación sexual y por ende el ejercicio de sus derechos. Esta implementación debe ir acompañada de un proceso sistemático de formación docente, preferentemente amparado en políticas educativas nacionales. 


\section{Referencias bibliográficas:}

Adams Tucker, L., George, G., Reardon, C., \& Panday, S. (2016). "Learning the basics": young people's engagement with sexuality education at secondary schools. Sex Education, 16(4), 337-352.

Allen, L. (2005). "Say everything": Exploring young people's suggestions for improving sexuality education. Sex Education, 5(4), 389404.

Allen, L. (2008). “They Think You Shouldn”t be Having Sex Anyway': Young People's Suggestions for Improving Sexuality Education Content. Sexualities, 11(5), 573-594.

Bandura, A. (2006). Guide for constructing self-efficacy scales. SelfEfficacy Beliefs of Adolescents, 5(307-337).

Bay-Cheng, L. Y. (2003). The trouble of teen sex: The construction of adolescent sexuality through school-based sexuality education. Sex Education: Sexuality, Society and Learning, 3(1), 61-74.

Carvajal, A. (24 de mayo de 2017). Lenín Moreno firma sus primeros 10 Decretos; elimina el Plan familia. El Comercio, pp.1-2. Recuperado de

http://www.elcomercio.com/actualidad/leninmoreno-decretosplanfamilia-buenvivir-presidencia.html

Chandra-Mouli, V., Svanemyr, J., Amin, A., Fogstad, H., Say, L., Girard, F., \& Temmerman, M. (2015). Twenty years after International Conference on Population and Development: where are we with adolescent sexual and reproductive health and rights? Journal of Adolescent Health, 56(1), S1-S6.
Coordinación General de Planificación y Gestión Estratégica del Ecuador (CGPGEE). (2016). Proyecto Plan Nacional de Fortalecimiento de la Familia. Recuperado de

http://www.planfamilia.gob.ec/wpcontent/uploads/downloads/2016/07/ PROYECTO-PLAN-FAMILIA-ECUADOR-MAYO-SIF.pdf

Darré, S., Jerves, E., Castillo, J., \& Enzlin, P. (2015). SEXUALITY EDUCATION IN LATIN AMERICA. Evidence-Based Approaches to Sexuality Education: A Global Perspective, 277.

Denno, D. M., Hoopes, A. J., \& Chandra-Mouli, V. (2015). Effective strategies to provide adolescent sexual and reproductive health services and to increase demand and community support. Journal of Adolescent Health, 56(1), S22-S41.

Forrest, S., Strange, V., Oakley, A., \& Ripple Study Team. (2004). What do young people want from sex education? The results of a needs assessment from a peer-led sex education programme. Culture, Health \& Sexuality, 6(4), 337-354.

Haberland, N., \& Rogow, D. (2015). Sexuality education: emerging trends in evidence and practice. Journal of Adolescent Health, $56(1), \mathrm{S} 15-\mathrm{S} 21$.

Helmer, J., Senior, K., Davison, B., \& Vodic, A. (2015). Improving sexual health for young people: making sexuality education a priority. Sex Education, 15(2), 158-171.

Hilton, G. L. S. (2003). Listening to the Boys: English boys' views on the desirable characteristics of teachers of sex education. Sex Education, 3(1), 33-45.

Hirst, J. (2008). Developing sexual competence? Exploring strategies for the provision of effective sexualities and relationships education. Sex Education, 8(4), 399-413. 
Instituto Nacional de Estadísticas y Censos del Ecuador (INEC). (2010). Resultados del Censo 2010 de población y vivienda en el Ecuador. Recuperado de http://www.ecuadorencifras.gob.ec/ censo-de-poblacion-y-vivienda/

Instituto Nacional de Estadísticas y Censos del Ecuador (INEC). (2014). Resultados Demografia y Salud Sexual y Reproductiva. Recuperado de http://www.ecuadorencifras.gob.ec/msp-einec-presentan-resultados-de-demografia-y-salud-sexual-yreproductiva/

International Planned Parenthood Federation (IPPF). (2006). Framework for Comprehensive Sexuality Education (CSE). Recuperado de http://www.ippf.org/sites/default/files/ippf_framework_for_ comprehensive_sexuality_education.pdf

International Planned Parenthood Federation (IPPF). (2012). From evidence to action: Advocating for comprehensive sexuality education. London . Recuperado de Http://Www. Ippf. Org/NR/ Rdonlyres/FB127CA3-4315-4959-BF99-F23BAB9F5AB4/0/ SexEdAdvocacy. Pdf (3 June.

Kirby, D. (2002). The impact of schools and school programs upon adolescent sexual behavior. Journal of Sex Research, 39(1), 27-33.

Linacre, J. M. (2002). Optimizing rating scale category effectiveness. $J$ Appl Meas, 3(1), 85-106.

Lora Rocha, Ó., Castro Mantilla, M. D., \& Salinas Mulder, S. (2009). Situación del embarazo en la adolescencia en la subregion andina.

Lupton, D., \& Tulloch, J. (1996). "All red in the face": students' views on school-based HIV/AIDS and sexuality education. The Sociological Review, 44(2), 252-271.
MacDonald, J.-A., Gagnon, A. J., Mitchell, C., Di Meglio, G., Rennick, J. E., \& Cox, J. (2011). Asking to listen: Towards a youth perspective on sexual health education and needs. Sex Education, 11(4), 443-457.

Matthias, R. E., Lubben, J. E., Atchison, K. A., \& Schweitzer, S. O. (1997). Sexual activity and satisfaction among very old adults: results from a community-dwelling Medicare population survey. The Gerontologist, 37(1), 6-14.

Measor, L. (2004). Young people's views of sex education: Gender, information and knowledge. Sex Education, 4(2), 153-166.

Ministerio de Educación del Ecuador (MEE). (2012). Archivo Maestro Instituciones Educativos. Recuperado de ew http://educacion. gob.ec/amie/.

Ministerio de Salud del Ecuador (MSE). (2012). Embarazo Adolescente y Fecundidad en Cifras. Recuperado de http://instituciones. msp.gob.ec/misalud/index.php?option=com_content\&view $=$ article $\&$ id $=668$ :embarazo-adolescente-y-fecundidad-encifras\&catid $=51:$ mi-salud-al-dia\&Itemid $=242$

Muhanguzi, F. K., \& Ninsiima, A. (2011). Embracing teen sexuality: Teenagers' assessment of sexuality education in Uganda. Agenda, 25(3), 54-63.

O'Higgins, S., \& Gabhainn, S. N. (2010). Youth participation in setting the agenda: learning outcomes for sex education in Ireland. Sex Education, 10(4), 387-403.

Ortiz, W., \& Palacios, M. D. (2011). Políticas y Curriculo para la educación sexual en los colegios de la ciudad de Cuenca. In M. Jerves, \& P. Enzlin, Resultados de las investigaciones (pp. 7-21). Cuenca: Grafisum. 
Pineda, E., \& Jerves, E. (2015). Percepciones y expectativas de los y las adolescentes sobre la educación sexual que reciben en los colegios de la ciudad de Cuenca.

Patton, G. C., Coffey, C., Cappa, C., Currie, D., Riley, L., Gore, F., Degenhardt, L., Richardson, D., Astone, N., Sangowawa, A. (2012). Health of the world's adolescents: a synthesis of internationally comparable data. The Lancet, 379(9826), 1665-1675.

Rijsdijk, L. E., Lie, R., Bos, A. E., Leerlooijer, J. N., \& Kok, G. (2013). Sexual and reproductive health and rights: implications for comprehensive sex education among young people in Uganda. Sex Education, 13(4), 409-422.

Santelli, J., Ott, M. A., Lyon, M., Rogers, J., Summers, D., \& Schleifer, R. (2006). Abstinence and abstinence-only education: a review of US policies and programs. Journal of Adolescent Health, $38(1), 72-81$.

Shoveller, J. A., Johnson, J. L., Langille, D. B., \& Mitchell, T. (2004). Socio-cultural influences on young people's sexual development. Social Science \& Medicine, 59(3), 473-487.

Simovska, V. \& Kane, R. (2015). Sexuality education in different contexts: limitations and possibilities. Health Education, 115(1), 2-6.

Suellentrop, K. (2011). What works 2011-2012: curriculum-based programs that help prevent teen pregnancy. Washington, DC: National Campaign to Prevent Teen and Unplanned Pregnancy; 2011. Recuperado de Thenationalcampaign. Org/Resource/What-Works.

Thomas, F., \& Aggleton, P. (2016). School-Based Sex and Relationships Education: Current Knowledge and Emerging Themes. In Global Perspectives and Key Debates in Sex and Relationships Education: Addressing Issues of Gender, Sexuality, Plurality and Power (pp. 13-29). Springer.
UNESCO. (2015). Emerging evidence, lessons and practice in ComprehensiveSexualityEducation.Aglobalreview.Recuperado de http://unesdoc.unesco.org/images/0024/002431/243106e. pdf

UNESCO, U. (2009). International Technical Guidance on Sexuality Education. An evidence-informed approach for schools, teachers and health educators. Recuperado de http://unesdoc. unesco.org/images/0018/001832/183281e.pdf

UNFPA. (2014). Comprehensive Sexuality Education. Recuperado de http://www.unfpa.org/comprehensive-sexuality-education

Viveros, M. (2010). La sexualización de la raza y la racialización de la sexualidad en el contexto latinoamericano actual.

Walters, A. S., \& Hayes, D. M. (2007). Teaching about sexuality: Balancing contradictory social messages with professional standards. American Journal of Sexuality Education, 2(2), 27 49

World Health Organization (WHO). (2010). Standards for sexuality education in Europe: a framework for policy makers, educational and health authorities and specialists. Cologne: Federal Centrefor Health Education, BZgA. 